\title{
ENFERMEIROS DE SERVIÇOS DE URGÊNCIA E EMERGÊNCIA PSIQUIÁTRICA: ANÁLISE DE PERFIL PROFISSIONAL E EDUCACIONAL
}

\author{
Divane de $\operatorname{Vargas}^{1}$, Janaina Soares ${ }^{2}$, Talita Dutra Ponce ${ }^{3}$, Bruna Batista de Oliveira ${ }^{4}$
}

\begin{abstract}
RESUMO: O estudo caracterizou o perfil profissional e educacional de enfermeiros de urgência e emergência psiquiátrica, identificando sua associação com a prática clínica. Estudo transversal realizado com 184 enfermeiros, em 17 serviços de atendimento de urgências e emergências psiquiátricas no estado de São Paulo, entre fevereiro e outubro de 2012. Evidenciou-se associação significativa entre o perfil dos enfermeiros e a prática clínica no atendimento de urgências e emergência psiquiátrica e os maiores preditores dessa associação foram: sexo masculino $(\mathrm{OR}=9,05)$, tempo de profissão maior que 10 anos $(\mathrm{OR}=2,67)$ e ser egresso de instituições públicas de ensino $(O R=2,14)$. Enfermeiros do sexo masculino continuam sendo a linha de frente dos atendimentos em urgência e emergência psiquiátrica, sugerindo a perpetuação do modelo embasado na força física. A experiência profissional e maior carga horária durante a graduação são fatores que podem se constituir em melhor prática clínica em emergências psiquiátricas.

DESCRITORES: Enfermagem psiquiátrica; Psiquiatria; Prática Profissional; Cuidados de Enfermagem; Serviços de Emergência Psiquiátrica.
\end{abstract}

\section{PSYCHIATRIC URGENCY AND EMERGENCY CARE NURSES: AN ANALYSIS OF THEIR PROFESSIONAL AND EDUCATIONAL PROFILE}

\begin{abstract}
The present study characterized the professional and educational profile of nurses working at psychiatric urgency and emergency services, identifying its association with clinical practice. A cross-sectional study was carried out with 184 nurses in 17 psychiatric urgency and emergency healthcare services in the state of São Paulo from February to October 2012. A significant association was found between the profile of nurses and clinical practice in psychiatric urgency and emergency care, and the greatest predictors of this association were: male gender $(\mathrm{OR}=9.05)$, length of time in the profession longer than 10 years $(\mathrm{OR}=2.67)$, and having graduated at public educational institutions $(\mathrm{OR}=2.14)$. The prevalence of male nurses in psychiatric urgency and emergency care services suggests the perpetuation of the model based on physical strength. Professional experience and increased workload during undergraduate studies are factors that may lead to a better clinical practice in psychiatric emergency services.
\end{abstract}

DESCRIPTORS: Psychiatric Nursing; Psychiatry; Professional Practice; Nursing Care; Psychiatric Emergency Services.

\section{ENFERMEROS DE SERVICIOS DE URGENCIA Y EMERGENCIA PSIQUIÁTRICA: ANÁLISIS DE PERFIL PROFESIONAL Y EDUCACIONAL}

RESUMEN: El estudio caracterizó el perfil profesional y educacional de enfermeros de urgencia y emergencia psiquiátrica, identificando su asociación con la práctica clínica. Estudio transversal realizado con 184 enfermeros en 17 servicios de atención de urgencias y emergencias psiquiátricas del estado de São Paulo, entre febrero y octubre de 2012. Se evidenció asociación significativa entre el perfil de los enfermeros y la práctica clínica en atención de urgencias y emergencias psiquiátricas, los mayores predictores de esta asociación fueron: sexo masculino $(\mathrm{OR}=9,05)$, tiempo desde graduación superior a 10 años $(\mathrm{OR}=2,67)$ y ser egresado de instituciones públicas de enseñanza $(\mathrm{OR}=2,14)$. Los enfermeros de sexo masculino continúan constituyendo la primera línea de atención en urgencias y emergencias psiquiátricas, sugiriendo la continuidad del modelo basado en la fuerza física. La experiencia profesional y mayor carga horaria durante el curso de grado son factores que pueden constituirse en mejor práctica clínica en emergencias psiquiátricas.

DESCRIPTORES: Enfermería Psiquiátrica; Psiquiatría; Práctica Profesional; Atención de Enfermería; Servicios de Urgencia Psiquiátrica.

${ }^{1}$ Enfermeiro. Doutor em Ciências. Docente de Enfermagem da Universidade de São Paulo. São Paulo, SP, Brasil.

2Enfermeira. Doutora em Ciências. Docente de Enfermagem da Universidade de São Paulo. São Paulo, SP, Brasil.

${ }^{3}$ Enfermeira. Doutoranda em Enfermagem. Universidade de São Paulo. São Paulo, SP, Brasil.

${ }^{4}$ Discente de Enfermagem. Universidade de São Paulo. São Paulo, SP, Brasil.

Autor Correspondente:

Talita Dutra Ponce

Universidade de São Paulo

Av. Dr. Enéas de Carvalho Aguiar, 419 - 05403-000 - São Paulo, SP, Brasil

E-mail: talitadp@usp.br
Recebido: 16/02/2017

Finalizado: $18 / 10 / 2017$ 


\section{- INTRODUÇÃO}

O termo emergência psiquiátrica pode ser definido por perturbação aguda no comportamento, pensamento e/ou no humor em um paciente que, se não tratado, pode trazer danos tanto para ele mesmo quanto para terceiros, podendo ser considerado mais grave quando envolve risco iminente de vida, como por exemplo, nas tentativas de suicídio ${ }^{(1)}$.

Urgências e emergências psiquiátricas são comuns nos cenários de atendimentos especializados em psiquiatria e em serviços gerais de pronto atendimento, sendo quadros que demandam medidas imediatas durante o atendimento para evitar desfechos graves ${ }^{(2)}$.

Nos Estados Unidos, estima-se que a prevalência de transtornos mentais graves na população geral seja de $4,2 \%{ }^{(3)}$. No Brasil, foram encontrados altos índices de transtornos mentais na população adulta, entre 20 a 56\%, com destaque para populações específicas, como mulheres e trabalhadores ${ }^{(4)}$.

Pressupõe-se que índices elevados de transtornos mentais repercutam em altas taxas de atendimentos de urgências e emergências psiquiátricas. Na maioria das vezes, o enfermeiro e sua equipe são os profissionais que têm o primeiro contato com os pacientes nessas situações, exigindo desses trabalhadores a intervenção imediata, juntamente com a equipe multiprofissional, no intuito de evitar maiores prejuízos à saúde do indivíduo e eliminar possíveis riscos à sua vida ou a terceiros ${ }^{(5)}$.

Apesar da importância do atendimento adequado às pessoas em situação de urgências e emergências psiquiátricas, estudos vêm demonstrando que equipes de enfermagem encontram dificuldades de comunicação, falta de conhecimento e experiência na área, além de, no caso do enfermeiro, acúmulo de atividades ${ }^{(6-7)}$. Esses fatores, associados à estrutura física precária, falta de recursos humanos ${ }^{(8)} \mathrm{e} o$

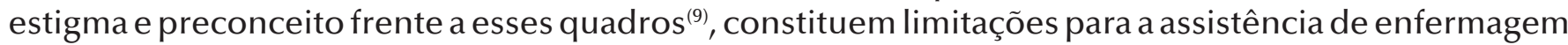
adequada aos pacientes.

Há poucos estudos disponíveis no Brasil sobre este fenômeno(10-11). Apesar de contribuírem para discussões, os mesmos são limitados a algumas regiões do país e se ocupam de traçar o perfil de trabalhadores de serviços gerais de urgência e emergência e aqueles de cunho psiquiátrico.

Assim, identificar o perfil dos enfermeiros que atuam em serviços de urgência e emergência psiquiátrica é relevante para a área de enfermagem psiquiátrica, pois, além de traçar um diagnóstico da situação, pode servir de subsídios para o planejamento de estratégias de formação e capacitação, visto que esses aspectos repercutem na assistência de enfermagem à pessoa em situação de urgência e emergência psiquiátrica, que, quando realizada com segurança, prontidão e qualidade, é capaz de determinar a aceitação e adesão do indivíduo atendido ao tratamento desse quadro ${ }^{(12)}$.

Considerando que a falta de conhecimento e experiência na área de enfermagem psiquiátrica tem se constituído como limitantes para a prática do enfermeiro em serviços de urgência e emergência dessa especialidade ${ }^{(6,8)}$, julgou-se oportuno realizar esse estudo, que objetiva caracterizar o perfil sociodemográfico, profissional e educacional de enfermeiros que trabalham em serviços de atendimento a urgência e emergência psiquiátrica, identificando sua associação com a prática clínica.

\section{MÉTODO}

Estudo transversal realizado em 17 serviços públicos, referenciados de atendimento à urgência e emergência psiquiátrica da cidade de São Paulo, que estavam em funcionamento no período da coleta realizada entre fevereiro e outubro de 2012.

Para coleta de dados, utilizou-se questionário contendo questões que abordavam as características sociodemográficas (sexo, estado civil e idade), as características profissionais (local de trabalho, tempo de profissão, turno de trabalho e a prática clínica no atendimento de urgência se emergência psiquiátricas - considerou-se como prática clínica a experiência e a frequência de atendimento em urgências e emergências psiquiátricas na prática profissional dos entrevistados) e educacionais (caráter da instituição de graduação, possuir pós-graduação tipo Lato/ Stricto senso, área de realização da pós-graduação, preparo durante a graduação em enfermagem para atuar em urgência e emergência 
psiquiátrica e tipo de preparo recebido).

Foram incluídos na amostra 184 enfermeiros de 17 serviços públicos de saúde, referenciados para o atendimento de urgência e emergência psiquiátrica no município de São Paulo, no período da coleta e que responderam afirmativamente à pergunta inicial "Você presta atendimento a quadros de urgência e emergência psiquiátrica em seu local de trabalho?". Foram excluídos do estudo enfermeiros que responderam negativamente a pergunta inicial $(n=53)$ e aqueles que estavam em período de férias ou em licença de qualquer natureza durante o período da coleta de dados.

A fim de possibilitar a participação representativa de enfermeiros dos três turnos de trabalho e das principais regiões do município, a coleta de dados foi realizada nos períodos da manhã, tarde e noite e em serviços referenciados da região sul, centro, leste, oeste e norte da cidade. Visando interferir o mínimo possível no andamento da rotina de trabalho dos profissionais, os instrumentos de coleta foram distribuídos sempre no final de cada turno, com a devolução dos mesmos preenchidos em um período de 24 a 48 horas e os participantes foram orientandos a não se identificarem nos instrumentos.

Foi realizada análise descritiva dos dados, os quais foram apresentados em número absolutos e percentagens, bem como média das variáveis contínuas. Para verificar associação entre o perfil dos trabalhadores e prática clínica em urgência e emergência psiquiátrica, foi utilizada uma análise de regressão logística. Inicialmente os dados foram submetidos à regressão logística univariada, para identificar as variáveis que influenciavam significativamente na prática clínica, como medidas para estimar o Odds Ratio (OR) com intervalo de confiança de 95\%.

Todas as variáveis que apresentaram valores de $p \leq 0,20$ (sexo, estado civil, idade, local de trabalho, tempo de profissão, turno de trabalho, instituição de formação, pós-graduação, tipo de pós-graduação, área de pós-graduação, preparo durante a graduação para atuar em emergências psiquiátricas e tipo de preparo recebido para atuar no atendimento a urgência e emergência psiquiátrica durante a graduação) foram introduzidas passo a passo no modelo de regressão logística múltipla. Nesse passo, os valores de $\mathrm{p} \leq 0,05$ foram considerados significantes. Todas as análises foram realizadas utilizando-se o software Statistical Package for the Social Sciences (SPSS), versão 22.0.

A pesquisa foi aprovada pelo Comitê de Ética em Seres Humanos da Secretaria Municipal de Saúde do município de São Paulo, com o parecer número 029/12.

\section{RESULTADOS}

Os enfermeiros que atuam em serviços de atendimento de urgências e emergências psiquiátricas caracterizaram-se por serem indivíduos do sexo feminino ( $n=140 / 76,1 \%)$, solteiros $(n=78 / 42,4 \%)$, com média de idade de 36,6 anos. Quanto às características profissionais, os indivíduos apresentavam tempo de profissão de 6 a 10 anos $(n=61 / 33,2 \%), 63(34,2 \%)$ informaram jornada de trabalho de 12 horas (turno integral), 70 (38\%) trabalham em unidades de internação psiquiátrica em hospitais gerais, $61(33,2 \%)$ em hospitais psiquiátricos e 53 (28,8\%) serviços de urgência e emergência psiquiátrica em hospitais gerais, sendo que todos esses serviços realizavam em suas rotinas atendimentos à urgência e emergência psiquiátrica.

Quanto ao perfil educacional, os enfermeiros com título de pós-graduação e que realizaram a graduação em instituições privadas de ensino foram a maioria, perfazendo 138 (75\%) e 126 (68,5\%) da amostra, respectivamente. A área de pós-graduação mais frequente entre os enfermeiros foi especialização em enfermagem médico-cirúrgica ( $n=42 / 22,8 \%)$, seguido por enfermagem psiquiátrica $(n=35 / 19)$. Enfermeiros informaram ter recebido preparo na área para atendimento em urgências e emergências psiquiátricas durante a graduação em enfermagem $(n=107 / 58,2 \%)$, sendo esse preparo oferecido por meio de aulas expositivas ( $n=80 / 43,5 \%$ ).

Um percentual significativo de enfermeiros $(n=53 / 22 \%)$, daqueles convidados a participar da pesquisa, informou não ter experiência no atendimento em urgências e emergências psiquiátricas, e, portanto, segundo os pressupostos desse estudo, negaram possuir prática clínica no atendimento das urgências e emergências psiquiátricas (Tabela 1). 
Tabela 1 - Descrição das respostas referentes à prática clínica (experiência e frequência de atendimento de urgências/ emergências psiquiátricas) dos participantes do estudo (N=184). São Paulo, SP, Brasil, 2017

\begin{tabular}{lcc} 
Prática Clínica & $\mathbf{N}$ & $\mathbf{\%}$ \\
\hline Experiência no atendimento de urgência e emergências psiquiátricas & & \\
\hline Sim & 184 & 100 \\
\hline Frequência de atendimento de urgência e emergências psiquiátricas na prática profissional & & \\
\hline Diariamente & 61 & 33,1 \\
\hline Semanalmente & 24 & 13 \\
\hline Mensalmente & 6 & 3,3 \\
\hline Raramente & 29 & 15,8 \\
\hline Não respondeu & 64 & 34,8 \\
\hline Total & 184 & 100
\end{tabular}

Na regressão logística univariada, não houve associação entre a prática clínica (experiência e frequência de atendimento de urgências e emergências psiquiátricas) e as variáveis: estado civil $(p=0,47)$, idade $(p=1,19)$, turno de trabalho $(p=0,50)$, pós-graduação $(p=0,38)$, tipo de pós $(p=1)$, preparo durante a graduação para atuar em emergências psiquiátricas $(p=0,3)$ e tipo de preparo recebido para atuar no atendimento a urgência e emergência psiquiátrica durante a graduação $(p=0,34)$.

Incluíram-se no modelo as variáveis que apresentaram significância estatística na análise univariada: sexo, local de trabalho, área de pós-graduação, tempo de profissão e instituição de graduação, as quais se mostraram associadas à prática clínica, conforme demonstrado na Tabela 2. Os resultados desta análise apontam que os maiores preditores dessa associação foram: ser do sexo masculino $(\mathrm{OR}=9,05)$, com tempo de profissão entre 11 e 20 anos $(\mathrm{OR}=2,67)$ e com graduação em instituições públicas de ensino $(\mathrm{OR}=2,14)$.

Tabela 2 - Relação entre a prática clínica (experiência e frequência de atendimento de urgências/emergências psiquiátricas) e as variáveis que apresentaram significância estatística na análise univariada (N=184). São Paulo, SP, Brasil, 2017

\begin{tabular}{lccc} 
Variáveis & OR & IC 95\% & P \\
\hline Sexo & & & 0 \\
\hline Feminino & 1 & & 0 \\
\hline Masculino & 9,05 & $(2,16-59,16)$ & 0 \\
\hline Local de Trabalho & & & 0 \\
\hline Serviço de emergência & 1 & & 0 \\
\hline Hospital Geral & 0,16 & $(0,04-0,54)$ & 0,07 \\
\hline Hospital Psiquiátrico & 0,27 & $(0,06-1,09)$ & 0 \\
\hline Área de Pós-graduação & & & 0,32 \\
\hline Psiquiátrico & 1 & & 0,09 \\
\hline Médico-cirúrgico & 0,44 & $0,08-2,16$ & 0,7 \\
\hline Saúde Pública & 0,22 & $0,03-1,26$ & 0,01 \\
\hline Administração & 0,69 & $0,10-4,60$ & \\
\hline Outros & 0,03 & $0,00-0,36$ & 0 \\
\hline Tempo de profissão & & & 0,01 \\
\hline Até 10 anos & 1 & & 0,39 \\
\hline De 11 a 20 anos & 2,67 & $(1,21-5,97)$ & 0 \\
\hline 21 anos ou mais & 1,5 & $(0,57-3,84)$ & 0,05
\end{tabular}


Ainda em relação ao tempo de profissão, o estudo encontrou associação estatisticamente significativa entre o tempo de profissão e a frequência do atendimento de urgências e emergências psiquiátricas $(p=0,04)$, enfermeiros com tempo de experiência entre 21 e 26 anos $(n=14 / 80 \%)$ informaram atender esses quadros diariamente, esse percentual cai para $(n=14 / 46,7 \%)$ para aqueles com tempo de profissão menor que cinco anos.

\section{- DISCUSSÃO}

Os enfermeiros que atuam em serviços de atendimento às urgências e emergências psiquiátricas do município de São Paulo são majoritariamente mulheres, adultas jovens, solteiras, e com tempo de profissão de 11 a 20 anos. Este resultado coincide com o perfil dos enfermeiros brasileiros ${ }^{(13)}$, que descreve que aproximadamente $90 \%$ do total de enfermeiros são do sexo feminino e que a enfermagem é uma das dez profissões da área de saúde que contribui para a feminização da força de trabalho no setor de saúde no país. Além disso, o fato da maioria dos profissionais pertencer ao estado civil solteiro pode estar relacionado a esses enfermeiros serem em sua maioria adultos jovens, com faixa etária abaixo dos 39 anos.

Em relação às características educacionais, 68,5\% dos profissionais entrevistados eram oriundos de instituições privadas de ensino, reflexo da oferta do ensino de graduação em enfermagem no país, em que $87,4 \%$ das instituições são de caráter privado ${ }^{(14)}$. A maioria dos enfermeiros possuía pósgraduação em áreas de conhecimentos da enfermagem que não aquelas relacionadas à enfermagem psiquiátrica. Esse resultado chama atenção principalmente pelo fato de que existe privação de conhecimentos dessa área, quando comparada às outras, no currículo do enfermeiro durante a graduação ${ }^{(15)}$. Consequentemente, é a pós-graduação na área que contribui mais efetivamente para o preparo do profissional para atuação nessa especialidade, o que incluiu o atendimento as urgências e emergências ${ }^{(16)}$.

O fato dos enfermeiros não optarem pela pós-graduação em psiquiatria, mesmo trabalhando em serviços de referência para urgências e emergências psiquiátricas, pode estar ligado ainda ao estigma e preconceito em relação aos pacientes portadores de transtorno mental, e talvez, este fato esteja associado à escassez de cursos de especialização em enfermagem psiquiátrica em algumas regiões do Brasil ${ }^{(16)}$. O estigma também influencia no momento de escolha da área de pós-graduação, levando o profissional a optar por outras áreas do conhecimento que não a especialização em psiquiatria, apesar de esta constituir-se num mercado de trabalho em expansão para a enfermagem na atualidade.

Os enfermeiros do estudo (58,3\%) relataram ter recebido preparo na área de urgência e emergência psiquiátrica durante a graduação. Ainda que existam críticas à quantidade e a qualidade do conhecimento específico em enfermagem psiquiátrica ofertado(15), esse ponto deve ser considerado positivo e devese a mudanças realizadas nas grades curriculares das instituições públicas e privadas de graduação em saúde. Essas mudanças se baseiam na II Conferência Nacional de Saúde Mental (17), que apontou a necessidade das instituições formadoras de profissionais de saúde introduzirem temas de saúde mental nas grades curriculares, bem como promover a obrigatoriedade de estágios acadêmicos nas redes públicas e privadas de saúde.

O estudo mostrou que os profissionais do sexo masculino têm nove vezes mais chances de executarem prática clínica em urgências/emergências psiquiátricas do que os do sexo feminino. Esse fato sugere que, apesar da enfermagem majoritariamente ser constituída por profissionais do sexo feminino, os serviços de urgência e emergência psiquiátrica, de maneira geral, são locais de trabalho onde enfermeiros do sexo masculino são encontrados com maior frequência, e a presença majoritária dos homens nesses espaços pode estar atrelada às características dos atendimentos realizados, que exigem dos profissionais maior esforço físico para sua execução(18).

Sobre esse resultado, os atendimentos aos pacientes psiquiátricos parecem ser vistos como atendimentos em que se faz necessário o uso da força física, associada ao sexo masculino. Entretanto, o uso exclusivo da força nos atendimentos de urgências e emergências psiquiátricas abre espaço para outros questionamentos, já que essa abordagem deve ser utilizada apenas em contenção física ou mecânica e em casos de última necessidade, após o uso de técnicas como a abordagem verbal ou as 
contenções químicas, por exemplo(19), as quais certamente independem de força física, mas sim do conhecimento e do preparo técnico especializado. Mesmo nessas técnicas, em que se pressupõe que o uso da forca física seja uma prerrogativa para a sua execução, não há impedimentos para que esse seja realizado por enfermeiras do sexo feminino, quando instrumentalizadas de preparo específico ${ }^{(20)}$.

Os enfermeiros com tempo de profissão entre 11 e 20 anos apresentaram duas vezes mais chances de confirmar sua prática clínica nos casos de urgências e emergências psiquiátricas do que os enfermeiros com tempo de experiência profissional menor que 10 anos de profissão. Esse fato pode estar associado à falta de experiência dos enfermeiros com menos tempo de serviço, o que pode resultar em dificuldades na identificação dos quadros de urgências e emergências psiquiátricas.

Esta hipótese parece encontrar respaldo nos resultados desse estudo que apontou que os enfermeiros com tempo de profissão superior a 20 anos também eram aqueles que relatavam maior frequência de atendimento diário de quadros de urgência e emergência psiquiátrica, enquanto enfermeiros com menos de cinco anos de profissão identificaram atendimentos diários com menor frequência, mesmo exercendo suas atividades no mesmo local dos enfermeiros com maior tempo de experiência. Especula-se então que esses resultados podem estar associados à pouca habilidade dos enfermeiros menos experientes e sem preparo específico na identificação desses quadros, mesmo atuando neles com frequência.

Consistente com esse resultado, também chama atenção o fato de que mais de $20 \%$ dos enfermeiros convidados a participar do estudo negou prestar atendimento em situações de urgências e emergências psiquiátricas, ainda que alocados em serviços de referência para o atendimento desses quadros, levando a pressupor a possibilidade de que, mesmo quando envolvidos no atendimento dessas situações, esses enfermeiros não a caracterizem como tal.

A dificuldade de identificação de quadros de urgências e emergências psiquiátricas por enfermeiros precisa ser levada em consideração, uma vez que a não identificação de um quadro pode resultar em assistência inadequada. Especula-se que uma das causas para esse fenômeno possa ser a falta de preparo em reconhecer tais situações, levando o enfermeiro a negar tal experiência mesmo quando está envolvido nela.

Nesse contexto, é importante pensar em estratégias de educação permanente para esses enfermeiros, pois, é sabido que enfermeiros assistenciais se beneficiam de atualizações no serviço sobre a temática saúde mental e psiquiatria ${ }^{(21)}$, o que permite sugerir que essas estratégias de atualizações sejam focadas em enfermeiros, com pouco tempo de experiência profissional, em atendimentos de urgência e emergência psiquiátricas.

Os resultados desse estudo mostraram que enfermeiros formados em instituições públicas de ensino têm duas vezes mais chances de relatar prática clínica em urgências e emergências psiquiátricas do que enfermeiros formados em outras instituições. Este fato pode estar relacionado à quantidade e a qualidade do ensino que vem sendo desenvolvido nas instituições públicas, as quais, de maneira geral, oferecem carga horária maior, incluindo estágios práticos, quando comparadas às de cunho privado ${ }^{(22)}$, atendendo às exigências de formação com as competências necessárias para o enfermeiro atuar no campo da enfermagem psiquiátrica e da atenção psicossocial(23), o que, em última análise, pode contribuir para maior percepção do enfermeiro dos quadros de urgências e emergências psiquiátricas.

Os enfermeiros que atuam em serviços de porta aberta possuem maior prática clínica do que aqueles que realizam seus atendimentos de urgências e emergências psiquiátricas nos hospitais psiquiátricos e unidades psiquiátricas em hospitais gerais. Isto está relacionado à própria finalidade desses espaços, o que justifica o maior contato e prática clínica dos enfermeiros que ali atuam, conferindo-lhes, portanto, maior experiência nesses atendimentos e facilitando a identificação desses quadros. A alta prevalência de urgências e emergências psiquiátricas em serviços de urgência/emergência pode estar relacionada à insuficiência/ineficiência da rede de atenção de saúde mental do município, onde os serviços extra hospitalares não absorvem a demanda existente, que acaba se concentrando nas emergências, muitas vezes, sem necessidade ${ }^{(24)}$.

Reforçando a importância da formação na área para atuação nos serviços de urgências e emergências psiquiátricas, este estudo encontrou associação estatística significativa entre a prática clínica e possuir pós-graduação em psiquiatria, ou seja, ter realizado essa especialização foi um preditor da prática clínica 
em urgências e emergências psiquiátricas. Consistente com esse resultado, estudo prévio ${ }^{(25)}$ realizado $^{2}$ em um serviço de urgência e emergência mostrou que a qualidade dos diagnósticos de enfermeiros psiquiátricos foi apropriada em $92 \%$ dos casos, quando comparados àqueles sem formação na área, observando-se também maior pertinência dos encaminhamentos realizados por esses especialistas, refletindo na melhora da satisfação do paciente com o atendimento de enfermagem. Nesse sentido, esse estudo reforça a importância da formação especializada em saúde mental e psiquiatria do profissional enfermeiro em serviços de urgência e emergência desta área.

Apesar da importância dos resultados trazidos por este estudo e seus avanços para a área de conhecimento, ele deve ser considerado no âmbito de suas limitações, dentre elas, se trata de uma amostra de enfermeiros que, apesar de pertencer ao maior município brasileiro, certamente não representa a diversidade que pode ser encontrada no país. Não se buscou comparar o conhecimento de enfermeiros de serviços de internação e urgência e emergência, especialistas em enfermagem psiquiátrica, com os enfermeiros que não possuem tal especialização, ou são especialistas em outras áreas de conhecimento, o que seria desejável para verificar, com maior confiabilidade, se a formação específica influencia no preparo desses profissionais para o atendimento dos quadros de urgências e emergências psiquiátricas.

Outro fator que pode ser apontado como limitante do estudo foi para as características do instrumento utilizado, o qual não possibilitava maior detalhamento das informações fornecidas, como por exemplo, não foi possível identificar com precisão a carga horária de preparo em saúde mental e psiquiatria relatada por esses enfermeiros durante a graduação em enfermagem.

\section{- CONCLUSÃo}

Os enfermeiros do sexo masculino continuam na linha de frente da prática clínica em urgências e emergências psiquiátricas, sugerindo perpetuação de um modelo assistencial baseado na força física, apesar das mudanças ocorridas no cenário de atenção nos últimos anos e da evolução das tecnologias de enfermagem para a intervenção nessas situações.

Esse estudo contribui para o reconhecimento do perfil dos enfermeiros que atuam nos serviços de as urgências e emergência psiquiátricas, permitindo mapeamento da atual situação educacional e profissional nesta área. Permite também, o reconhecimento da importância do preparo técnico especializado nessa área para atuação nesses serviços, pois, ainda que a experiência adquirida no trabalho possa assegurar algum preparo, a formação em especialização parece ser fator decisivo não só para maior habilidade de atuação, mas também para identificação e melhor desempenho nos atendimentos a esses quadros.

Foram levantados questionamentos em relação às estratégias de ensino de graduação na área de saúde mental e psiquiatria, sugerindo a necessidade de estudos futuros que se ocupem desta questão. O estudo sugere ainda que estratégias de educação e atualização na área de saúde mental e psiquiatria sejam realizadas nestes serviços, direcionando-os especialmente a enfermeiros em início de carreira e ou menor tempo de prática.

\section{- REFERÊNCIAS}

1. Bassuk EL, Birk AW, Editors. Emergency Psychiatry: Concepts, Methods, and Practices. $2^{\mathrm{a}}$ ed. New York: Plenum Press; 2013.

2. Mayrogiourgou P, Brüne M, Juckel G. The management of psychiatric emergencies. Dtsch Arztebl Int. [Internet] 2011;108(13) [acesso em 01 fev 2017]. Disponível: http://dx.doi.org/10.3238/arztebl.2011.0222.

3. National Institutes of Health (NIH). National Institute of Mental Health. Serious Mental IIIness Among U.S. Adults. [Internet] Rockville, MD: NIH; 2014 [acesso em 01 fev 2017]. Disponível: https://www.nimh.nih.gov/health/ statistics/prevalence/serious-mental-illness-smi-among-us-adults.shtml.

4. dos Santos EG, de Siqueira MM. Prevalence of mental disorders in the Brazilian adult population: a systematic 
review from 1997 to 2009. J. Bras. Psiquiatr. [Internet] 2010;59(3) [acesso em 01 fev 2017]. Disponível: http://dx.doi. org/10.1590/S0047-20852010000300011.

5. de Sousa FSP, da Silva CAF, Oliveira EN. Emergency psychiatric service in general hospitals: a retrospective study. Rev. esc. enferm. USP. [Internet] 2010;44(3) [acesso em 01 fev 2017]. Disponível: http://dx.doi.org/10.1590/ S0080-62342010000300035.

6. Paes MR, Maftum MA. Communication between nursing team and patients with mental disorder in an emergency service. Cienc Cuid Saúde. [Internet] 2013;12(1) [acesso em 01 fev 2017]. Disponível: http://dx.doi. org/10.4025/cienccuidsaude.v12i1.15830.

7. Buriola AA, Kantorski LP, Sales CA, Matsuda LM. Nursing practice at a psychiatric emergency service: evaluation using fourth generation assessment. Texto Contexto Enferm. [Internet] 2016;25(1) [acesso em 01 fev 2017]. Disponível: http://dx.doi.org/10.1590/0104-070720160004540014.

8. Cristiano CC, Hey AP, Montezeli JH, Sales WB, Visentin A, Kaled M. Portador de transtorno mental em situação de emergência: Dificuldades de atendimento percebidas pela equipe de enfermagem em uma unidade mista. Cad. da Esc. de Saúde. [Internet] 2015;2(14) [acesso em 01 fev 2017]. Disponível: http://revistas.unibrasil.com.br/ cadernossaude/index.php/saude/article/view/225.

9. Elias ADS, Tavares CMM, Cortez EA. Impact of stigma of madness on the attention of nursing to psychiatric patient in emergency situation. Cienc Cuid Saúde. [Internet] 2013;12(4) [acesso em 01 fev 2017]. Disponível: http:// periodicos.uem.br/ojs/index.php/CiencCuidSaude/article/view/22553.

10. Cavalcante AKCB, Amorim PHC, Santos LN. Perfil da equipe de enfermagem no serviço de urgência e emergência em um hospital público de Teresina. R. Interd. [Internet] 2014;7(2) [acesso em 01 fev 2017]. Disponível: http://revistainterdisciplinar.uninovafapi.edu.br/index.php/revinter/article/view/401.

11. Avelino FVSD, Leite ARF, Fernandes MA, Avelino FPD, Madeira MZA, de Sousa LEN. Stress in nurses sector of emergency and emergency. Rev Enferm UFPI. [Internet] 2013;2(3) [acesso em 01 fev 2017]. Disponível: http:// www.ojs.ufpi.br/index.php/reufpi/article/view/974.

12. Kondo EH, Vilella JC, Borba LO, Paes MR, Maftum MA. A nursing team's approach to users of a mental health emergency room. Rev. esc. enferm. USP. [Internet] 2011;45(2) [acesso em 01 fev 2017]. Disponível: http://dx.doi. org/10.1590/S0080-62342011000200028.

13. Wermeliger M, Machado MH, Tavares MFL, de Oliveira ES, Moysés NMN. Análise da força de trabalho do setor saúde do Brasil: focalizando a feminilização. [Internet] Rio de Janeiro (RJ): Observatório de Recursos Humanos; 2006 [acesso em 01 fev 2017]. Disponível: http://www.ensp.fiocruz.br/observarh/arquivos/A\%20Forca\%20de\%20 Trabalho\%20do\%20Setor\%20de\%20Saude\%20no\%20Brasil\%20.pdf.

14. Ministério da Educação (BR). Secretaria Executiva. Instituto Nacional de Estudos e Pesquisas Educacionais Anísio Teixeira. Censo da educação superior 2012: resumo técnico. Brasília: Instituto Nacional de Estudos e Pesquisas Educacionais Anísio Teixeira; 2014.

15. Magnago C, Tavares CMM. Nursing training focusing on ideals of the psychiatric reform. Rev Enferm Brasil. 2011;10(2):99-107.

16. Scochi CGS, Munari DB, Gelbcke FL, Erdmann AL, Gutiérrez MGR, Rodrigues RAP. The Strict Sense Nursing postgraduation in Brazil: advances and perspectives. Rev. bras. enferm. [Internet] 2013;66(n.esp) [acesso em 01 fev 2017]. Disponível: http://dx.doi.org/10.1590/S0034-71672013000700011.

17. Ministério da Saúde (BR). Secretaria de Assistência à Saúde. Departamento de Assistência e Promoção à Saúde. Coordenação de Saúde Mental. Relatório final da $2^{a}$ Conferência Nacional de Saúde Mental. Brasília: Ministério da Saúde; 1994.

18. de CarvalhoMB, Felli VEA. Psychiatric nursing work and workers' health problems. Rev. Latino-Am. Enfermagem. [Internet] 2006;14(1) [acesso em 01 fev 2017]. Disponível: http://dx.doi.org/10.1590/S0104-11692006000100009.

19. Conselho Federal de Medicina (CFM). Resolução CFM n. 2.057/2013, de 20 de setembro de 2013. Normatiza o atendimento médico a pacientes portadores de transtorno mental. [Internet] Brasília; 2013 [acesso em $01 \mathrm{fev}$ 2017]. Disponível: http://www.portalmedico.org.br/resolucoes/CFM/2013/2057_2013.pdf. 
20. Conselho Federal de Enfermagem (COFEN). Resolução COFEN n. 427/2012, de 7 de maio de 2012 (BR). Normatiza os procedimentos da enfermagem no emprego de contenção mecânica de pacientes. [Internet] Brasília; 2012 [acesso em 01 fev 2017]. Disponível: http://www.cofen.gov.br/resoluo-cofen-n-4272012_9146.html.

21. Ikuta CY, dos Santos MA, Badagnan HF, Donato ECSG, Zanetti ACG. Nursing workers' knowledge in psychiatric emergency situations: an integrative review. Rev. Eletr. Enf. [Internet] 2013;15(4) [acesso em $01 \mathrm{fev}$ 2017]. Disponível: http://dx.doi.org/10.5216/ree.v15i4.20954.

22. Vargas D, Lenate JS, Bittencourt MN, Pereira CF. O ensino de Enfermagem em Saúde Mental e Psiquiátrica no Brasil: Análise das estruturas curriculares. No prelo 2017.

23. Magnago C, Tavares CMM. Psychiatric nursing education at Public Universities in the State of Rio de Janeiro. Rev. Eletr. Enf. [Internet] 2011;14(1) [acesso em 01 fev 2017]. Disponível: http://www.revenf.bvs.br/scielo. php?script=sci_arttext\&pid=S1518-19442012000100006.

24. Barros REM, Tung TC, Mari JJ. Psychiatric emergency services and their relationships with the mental health network in Brazil. Rev. Bras. Psiquiatr. [Internet] 2010;32(2) [acesso em 01 fev 2017]. Disponível: http://dx.doi. org/10.1590/S1516-44462010000600003.

25. Sinclair L, Hunter R, Hagen S, Nelson D, Hunt J. How effective are mental health nurses in A\&E departments? Emerg Med J. [Internet] 2006;23(9) [acesso em 01 fev 2017]. Disponível: http://dx.doi.org/10.1136/emj.2005.033175. 